\title{
Curvilinear 3-D Imaging Using Row-Column Addressed 2-D Arrays with a Diverging Lens: Phantom Study
}

Bouzari, Hamed; Engholm, Mathias; Beers, Christopher; Stuart, Matthias Bo; Nikolov, Svetoslav Ivanov; Thomsen, Erik Vilain; Jensen, Jørgen Arendt

Published in:

IEEE Transactions on Ultrasonics, Ferroelectrics and Frequency Control

Link to article, DOI:

10.1109/TUFFC.2018.2836384

Publication date:

2018

Document Version

Peer reviewed version

Link back to DTU Orbit

Citation (APA):

Bouzari, H., Engholm, M., Beers, C., Stuart, M. B., Nikolov, S. I., Thomsen, E. V., \& Jensen, J. A. (2018). Curvilinear 3-D Imaging Úsing Row-Column Addressed 2-D Arrays with a Diverging Lens: Phantom Study. IEEE Transactions on Ultrasonics, Ferroelectrics and Frequency Control, 65(7), 1182-1192. https://doi.org/10.1109/TUFFC.2018.2836384

\section{General rights}

Copyright and moral rights for the publications made accessible in the public portal are retained by the authors and/or other copyright owners and it is a condition of accessing publications that users recognise and abide by the legal requirements associated with these rights.

- Users may download and print one copy of any publication from the public portal for the purpose of private study or research.

- You may not further distribute the material or use it for any profit-making activity or commercial gain

- You may freely distribute the URL identifying the publication in the public portal 


\title{
Curvilinear 3-D Imaging Using Row-Column Addressed 2-D Arrays with a Diverging Lens: Phantom Study
}

\author{
Hamed Bouzari, Member, IEEE, Mathias Engholm, Christopher Beers Member, IEEE, \\ Matthias Bo Stuart, Member, IEEE, Svetoslav Ivanov Nikolov, Senior Member, IEEE, Erik Vilain Thomsen, \\ and Jørgen Arendt Jensen, Fellow, IEEE
}

\begin{abstract}
A double-curved diverging lens over the flat rowcolumn-addressed (RCA) 2-D array can extend its inherent rectilinear 3-D imaging field-of-view (FOV) to a curvilinear volume region, which is necessary for applications such as abdominal and cardiac imaging. Two concave lenses with radii of $12.7 \mathrm{~mm}$ and $25.4 \mathrm{~mm}$ were manufactured using RTV664 silicone. The diverging properties of the lenses were evaluated based on simulations and measurements on several phantoms. The measured FOV for both lenses in contact with tissue mimicking phantom were less than $15 \%$ different from the theoretical predictions, i.e., a curvilinear FOV of $32^{\circ} \times 32^{\circ}$ and $24^{\circ} \times 24^{\circ}$ for the $12.7 \mathrm{~mm}$ and $25.4 \mathrm{~mm}$ radii lenses. A synthetic aperture imaging sequence with single element transmissions was designed for imaging down to $140 \mathrm{~mm}$ at a volume rate of $88 \mathrm{~Hz}$. The performance was evaluated in terms of signal-to-noise ratio (SNR), FOV, and full-width-at-half-maximum (FWHM) of a focused beam. The penetration depths in a tissue mimicking phantom with $0.5 \mathrm{~dB} /(\mathrm{cm} \mathrm{MHz})$ attenuation were $100 \mathrm{~mm}$ and $125 \mathrm{~mm}$ for the lenses with radii of $12.7 \mathrm{~mm}$ and $25.4 \mathrm{~mm}$. The azimuth, elevation, and radial FWHM at $43 \mathrm{~mm}$ depth were $\mathbf{5 . 8}$, $5.8,1) \lambda$ and $(6,6,1) \lambda$. The results of this study confirm that the proposed lens approach is an effective method for increasing the FOV, when imaging with RCA 2-D arrays.
\end{abstract}

\section{INTRODUCTION}

An $N \times N$ element 2-D array can be operated utilizing only $2 N$ connections, when a row-column, cross-electrode, or toporthogonal-to-bottom-electrode (TOBE) addressing scheme is used [1]-[7]. This is contrary to the $N^{2}$ connections needed, when fully addressing the elements. In general, a row-columnaddressed (RCA) array is a 2-D matrix array, which is addressed via its row and column indices. Effectively, it consists of two 1-D arrays arranged orthogonal to each other. As an example, for a $256+256$ RCA array, a 2-D matrix array of equivalent size would have 65,536 elements, over a factor of 7 more than the current state-of-the-art X6-1 PureWave xMATRIX probe from Phillips (Eindhoven, Netherlands) having 9,212 elements [8]. This exhibits the potential of having very large RCA 2-D arrays with a low channel count and real-time capabilities.

H. Bouzari*, M. B. Stuart, and J. A. Jensen are with the Center for Fast Ultrasound Imaging, Department of Electrical Engineering, Technical University of Denmark, 2800 Kgs. Lyngby, Denmark.

M. Engholm, and E. V. Thomsen are with the Department of Micro- and Nanotechnology, Technical University of Denmark, 2800 Kongens Lyngby, Denmark.

C. Beers is with Sound Technology Inc, Analogic Ultrasound Group, PA16803, USA.

S. I. Nikolov is with BK Ultrasound ApS, 2730 Herlev, Denmark.

${ }^{*}$ e-mail of corresponding author is: bouzari@elektro.dtu.dk
It has been demonstrated in several studies that row-column technology is a realistic alternative to the state-of-the-art matrix probes, especially as a low-cost alternative. However, two major issues with the RCA arrays is that they can only emit acoustic energy directly in front of the array in a cross-shape to the sides, and only one-way focusing is possible in each lateral direction. Therefore the contrast and spatial resolutions are compromised compared with fully-addressed arrays of the same physical size. The contrast and spatial resolutions can be compensated for by using an apodization scheme, as well as increasing the size of the array [9]. However, imaging can only be performed in a rectilinear region in front of the array. For cardiac imaging, it is relevant to have a probe with a small foot-print capable of phased array imaging, such that the heart can be visualized through the ribs. True volumetric phased array imaging is possible with RCA arrays, provided that the array is double curved to spread the energy during transmit [2]. In [10] it was shown how to make a curved transducer element by bending the capacitive micromachined ultrasonic transducer (CMUT) RCA array in one dimension. However, manufacturing double curved transducer elements in two dimensions is challenging for both CMUT and piezoelectric transducer technologies. Another approach to spread the acoustic energy is by using a double curved diverging acoustic lens on top of the RCA array [11], [12]. Using a lens makes it easier to fabricate curved arrays, as it is not needed to manufacture curved elements, and also making a lens is a well-tested technology. An in-depth study of the possibilities in this approach has been previously investigated based on simulations by the authors [12].

In this paper, a more detailed discussion on the pros and cons of using a diverging lens based on measurements on phantoms is presented. The curvilinear volumetric imaging performance of an RCA array equipped with two mountable diverging lenses is investigated in terms of field-of-view (FOV), spatial and contrast resolution, as well as the SNR measurements using synthetic aperture imaging (SAI) technique. Initial results of this study have been published as a conference proceeding [13].

The paper is organized as follows: In the following Section II, the lens parameters are explained. Section III presents the utilized SAI sequence, a detailed overview of the measurements setup, as well as the quality assessment measures. Section IV explains and discusses the measurement results. The final Section concludes the paper. 


\section{Double-Curved Diverging Acoustic Lens}

Using a double-curved RCA 2-D array extends the volumetric imaging FOV of a flat RCA 2-D array to a curvilinear region. To spread the acoustic energy of a line-element curvilinearly along its larger dimension, it has to be curved like an arc to generate a diverging wave. Another approach is to use a double-curved diverging acoustic lens on top of the flat RCA array as shown in Fig. 1. The red dashed lines are illustrating the wave fronts at different time instances. A beamforming approach was proposed in [12] to accurately calculate the correct time-of-flights of the wave fronts using a diverging lens, and hence avoiding geometrical distortions. However, for the proposed beamfoming approach, the curvature of the wave fronts has to be estimated based on the physical properties of the lens in contact with the imaging medium. In this section a lens model adapted from optics into acoustics will be described. To simplify this model, the reflection from the boundaries inside the lens as well as the attenuation effects are neglected.

Given an arc or spherical cap with known width and height as shown in Fig. 1, the formulas relating the arc radius to its height and its base length are:

$$
\begin{gathered}
R=\frac{H}{2}+\frac{W^{2}}{8 H}, \\
0 \leq W \leq 2 R, \\
0 \leq H \leq R,
\end{gathered}
$$

where $W$ is the length of the chord defining the base of the arc, and $H$ is the height measured at the midpoint of the arc's base. In an arc with fixed radius, the largest base corresponds to a chord height of $H=W / 2=R$.

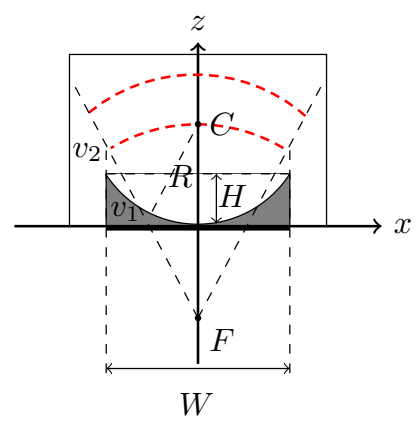

(a) Concave lens, $v_{1}<v_{2}$

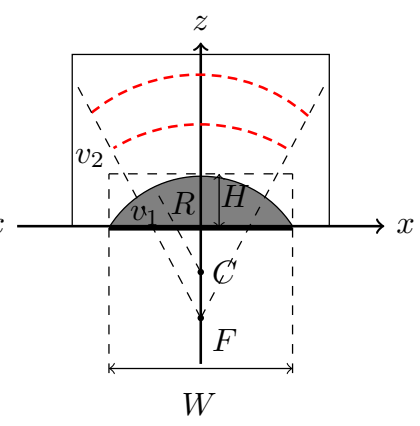

(b) Convex lens, $v_{1}>v_{2}$
Figure 1. A diverging lens with a material that has a constant speed of sound, $v_{1}$, can be manufactured in (a) concave or (b) convex shapes.

A concave diverging lens can be designed with a material, which has lower speeds of sound compared to the human tissue, as illustrated in Fig. 1a. It will have a higher thickness around the corners and the sides of the array, and less thickness close to center of the array. Alternatively, a convex diverging lens can be made from a material with a higher speed of sound compared to the human tissue, which is preferred for a better contact surface, as illustrated in Fig. 1b. However, due to the materials available for a lens and their speeds of sound, a concave lens was implemented in this study.
For a thin concave lens, where the radius is much larger than the arc height, the focal distance can be estimated from (2), which is the first-order Taylor expansion of the total surface power, i.e., $P=1 / F$, of the lens at its both flat and spherical surfaces [14]:

$$
\frac{1}{F} \approx\left(1-\frac{v_{1}}{v_{2}}\right) \frac{1}{R},
$$

where $v_{1}$ and $v_{2}$ are the speed of sound in the lens material and the medium, respectively. Depending on the application, the medium can be water, human soft tissue, or any other material. The focal length of the lens is indicated by $F$ and the radii of the arc is indicated by $R$. To have a larger curvature, i.e., $1 / R$, the ratio $v_{1} / v_{2}$ has to increase, and the curvature of the lens and the diverged wave fronts are exactly the same, when $v_{1} / v_{2}=2$. A positive value of the focal length indicates that the lens is converging the wave fronts and a negative value indicates that it is diverging. In a similar way, for a convex thin lens:

$$
\frac{1}{F} \approx\left(1-\frac{v_{2}}{v_{1}}\right) \frac{1}{R} .
$$

The f-number, $f_{\#}$, and angular FOV of the lens can be defined as:

$$
\begin{gathered}
f_{\#}=\frac{F}{W}, \\
\mathrm{FOV}=2 \cot ^{-1}\left(2 f_{\#}\right) .
\end{gathered}
$$

The theoretical $f_{\#}$ is between $0<f_{\#}<\infty$, which corresponds to $\pi>$ FOV $>0$. The terms "FOV" and "angular FOV" are used interchangeably throughout the manuscript. An angular FOV of zero means no divergence is occurring and only the rectilinear region in front of the array can be imaged, similar to flat RCA 2-D arrays. To increase the FOV, i.e. decreasing the $f_{\#}$, either the curvature of the lens or the ratio, e.g., $\left(1-v_{1} / v_{2}\right)$, in (2) has to increase. However, increasing the curvature of the lens, i.e., $1 / R$, corresponds to a smaller radius, which has a larger thickness for a fixed chord length. In practice, the lens chord, $W$, and its thickness, $H$, are both limited by the arrays aperture size as well as the attenuation through the lens. Therefore, to increase the FOV, the ratio $\left(1-v_{1} / v_{2}\right)$ has to increase. However, due to reflections of the pressure waves inside the lens, which are caused by the large acoustic impedance differences of the materials, lower $f_{\#}$ values are less feasible using a single lens. To minimize the reflection and the attenuation through the lens, a compounded lens made out of two or more different materials, but with lower acoustic impedance differences, could be made. This is, however, beyond the scope of this study.

\section{METHODS}

A SAI sequence is designed for imaging down to $140 \mathrm{~mm}$ of depth. It utilizes single element transmissions on the row elements, and the echoes are collected with all the column 
Table I

TRANSDUCER AND LENSES PARAMETERS AND SETUP CONFIGURATION

\begin{tabular}{llc}
\hline Center frequency & 3 & $\mathrm{MHz}$ \\
Pitch row & 270 & $\mu \mathrm{m}$ \\
Pitch column & 270 & $\mu \mathrm{m}$ \\
Number of rows & 62 & - \\
Number of columns & 62 & - \\
Pulse repetition frequency & 5 & $\mathrm{kHz}$ \\
No. of active elements in Tx & 1 & - \\
Scan depth (max range) & 14 & $\mathrm{~cm}$ \\
Emission center frequency & 3 & $\mathrm{MHz}$ \\
Sinusoid emission cycles & 2 & - \\
Focus in receive & Dynamic & - \\
Synthetic Tx apodization & Hann. & - \\
Rx electronic apodization & Hann. & - \\
Sampling frequency & 70 & $\mathrm{MHz}$ \\
Tx voltage & \pm 75 & $\mathrm{~V}$ \\
Lens $f_{\#}$ & $-1.5,-2.65$ & - \\
Sound speed in $20^{\circ} \mathrm{C}$ water & 1482 & $\mathrm{~m} / \mathrm{s}$ \\
Sound speed in vivo & 1540 & $\mathrm{~m} / \mathrm{s}$ \\
RTV664 silicone sound speed & 1000 & $\mathrm{~m} / \mathrm{mm}$ \\
RTV664 longitudinal atten. $($ at $3 \mathrm{MHz})$ & 1.4 & \\
\hline & & - \\
\hline
\end{tabular}

elements. For a speed of sound of $1540 \mathrm{~m} / \mathrm{s}, 182 \mu \mathrm{s}$ is required to acquire a single image line to a depth of $14 \mathrm{~cm}$ including penetrating the lens. For 62 emissions this is equivalent to a volume rate of $88 \mathrm{~Hz}$, and is the same sequence used in the simulation study in [12]. Hilbert transformed RF data are used for beamforming a low-resolution volume for every emission and finally, by summing all the low-resolution volumes in phase, a high-resolution volume is generated.

The transducer parameters of a PZT RCA 62+62 element 2-D array, as well as the imaging setup configuration, are shown in Table I [9]. The active layer of the PZT RCA array consists of a 1-3 composite of high-dielectric PZT-5H and epoxy. A metal stack of titanium tungsten, nickel vanadium, and gold in that order were then sputtered on the top and bottom surfaces of the composite for electrodes. Row and column elements were defined by scribing the top electrode in one direction and scribing the bottom electrode in the orthogonal direction [15]. Thereby, transmitting with row elements and receiving with column elements should image exactly the same volumetric region as transmitting with column elements and receiving with row elements. Thus, no preference is considered in transmitting with row elements and receiving the echoes with column elements, or vice versa [12]. The probe is connected to the experimental ultrasound scanner SARUS [16]. The measured Hilbert transformed RF signals are beamformed using a MATLAB (MathWorks Inc., Massachusetts, USA) implementation of the delay-and-sum (DAS) beamformer specific to curved RCA arrays [12]. To remove the otherwise apparent secondary echoes originating from the either ends of line-elements, roll-off apodization regions are placed at both ends of each element [5], [17]. The length of each apodization

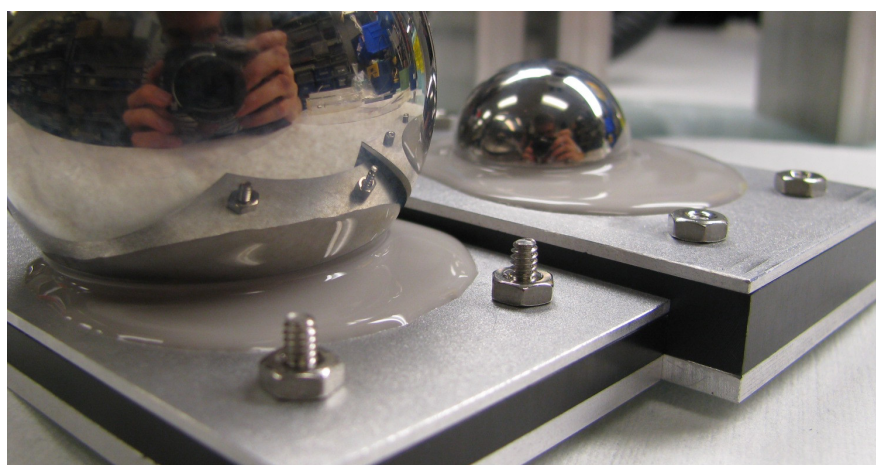

(a)

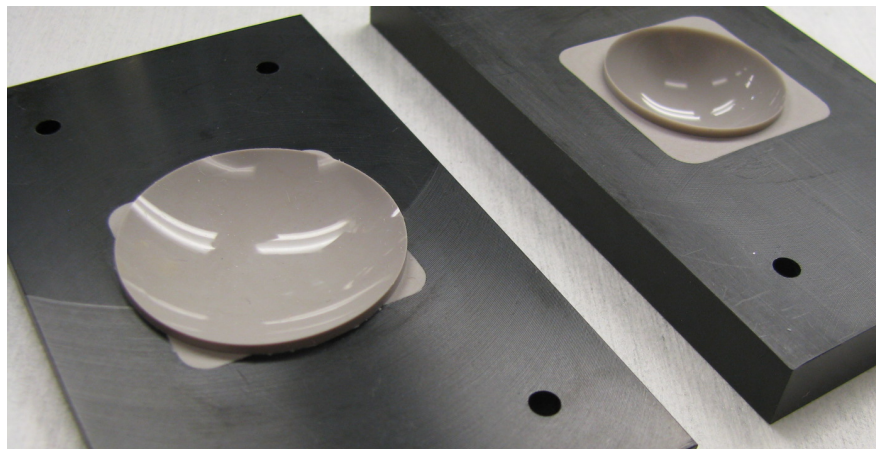

(b)

Figure 2. Illustration of the fabrication process for two concave diverging lenses made out of RTV664 silicone casted in a thermoplastic frame: (a) mold assembly and (b) final add-on lenses.

region was equal to 15 times the pitch of the array.

The add-on lenses were made by casting room temperature vulcanization (RTV) silicone, RTV664 (Momentive Performance Materials Inc., New York, USA ), into a rigid plastic frame and using a stainless steel ball bearing to form the curved surface. A mold assembly was made, consisting of a flat bottom plate and a top plate with a circular hole in which a steel ball sat during curing. The frame was sandwiched between these two plates, which were screwed together to ensure a controlled thickness. The silicone was poured into the mold assembly and degassed under vacuum to eliminate bubbles. During cure, the mold assembly was placed into a spring-loaded clamping fixture to press the ball into the cavity. Fig. 2a shows the steel balls sitting in the top plate after the RTV664 silicone cured but before the balls were removed from the molded parts.

Fig. 3 illustrates the schematics of two concave lenses with radii of $25.4 \mathrm{~mm}$ and $12.7 \mathrm{~mm}$ made out of RTV664 silicone, which has a lower speed of sound $(1000 \mathrm{~m} / \mathrm{s})$ compared to human soft tissue $(1540 \mathrm{~m} / \mathrm{s})$ and therefore follows the design shown in Fig. 1a. The defocusing aperture as well as the height of the spherical cap for the $25.4 \mathrm{~mm}$ radius lens are $27.31 \mathrm{~mm}$ and $3.98 \mathrm{~mm}$, and for the $12.7 \mathrm{~mm}$ radius lens they are $23.56 \mathrm{~mm}$ and $7.96 \mathrm{~mm}$. The minimum thickness at the center of both lenses is $0.75 \mathrm{~mm}$. The chord of the $25.4 \mathrm{~mm}$ radius lens is $31.75 \mathrm{~mm}$, but because of the thermoplastic frame, the minimum defocusing aperture scales down to $27.31 \mathrm{~mm}$. The $12.7 \mathrm{~mm}$ radius lens has a larger thickness, and the attenuation through the lens is higher. The longitudinal attenuation coefficient in the RTV664 silicone is approximately 


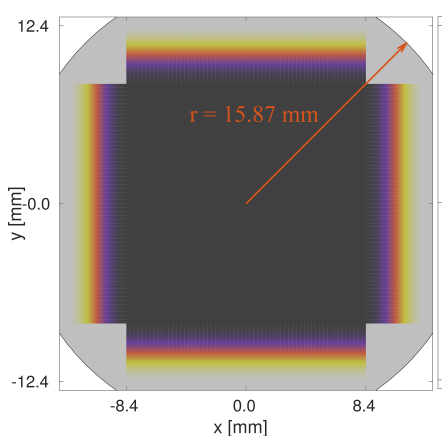

(a) (b)

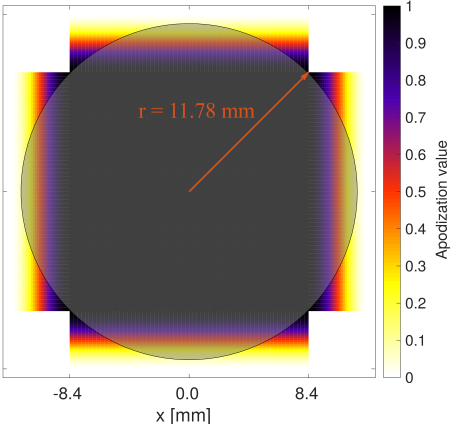

Figure 3. The figure illustrates the overlaid lenses on the array as well as the roll-off apodization regions in color gradient, (a) for lens with radius $25.4 \mathrm{~mm}$ and (b) for lens with radius $12.7 \mathrm{~mm}$. The chord radius of each lens is indicated by an orange arrow.

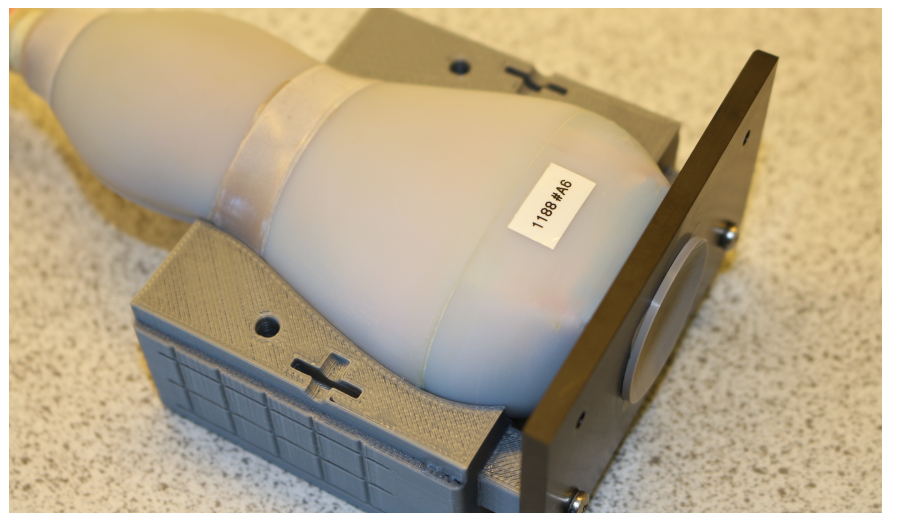

(a)

Figure 4. The lens modules are placed in front of the probe using a holder (half of the holder is shown in the figure).

$1.4 \mathrm{~dB} / \mathrm{mm}$ at $3 \mathrm{MHz}$. That corresponds to an axial attenuation of $6.62 \mathrm{~dB}$ for the $25.4 \mathrm{~mm}$ radii lens at its largest thickness at the corners, and it is $12.19 \mathrm{~dB}$ for the $12.7 \mathrm{~mm}$ radii lens. The lenses shown in Fig. $2 b$ are placed in front of the probe using a holder as shown in Fig. 4. The concave cavity of the lenses are filled with ultrasound gel.

To evaluate the imaging performance of both lenses, several ultrasound phantoms are used. An iron needle with diameter of $300 \mu \mathrm{m}$ facing towards the transducer along its central axis, was used as a point target in a water bath for characterizing the C-plane of the 3-D point spread function (PSF). To evaluate the full-width at half-maximum (FWHM) and the cystic resolution (CR) [18] as a function of depth, a geometrical copper wire phantom was used as line targets, where wires were located parallel to each other along the elevation plane with $10 \mathrm{~mm}$ spacing in both the axial and azimuth planes.

A tissue mimicking phantom with cylindrical anechoic targets, model 571 from Danish Phantom Design (Frederikssund, Denmark) with an attenuation of $0.5 \mathrm{~dB} /(\mathrm{cm} \mathrm{MHz})$ was used for signal-to-noise ratio (SNR) and contrast measurements.

The transmit pressure measurements of the lenses were carried out using the AIMS III intensity measurement system (Onda Corporation, Sunnyvale, California, USA) connected to the experimental research scanner SARUS. The OptiSon
Table II

THEORETICAL, SIMULATED, AND MEASURED FOVS OF BOTH LENSES

\begin{tabular}{|c|c|c|c|}
\hline \multirow{2}{*}{$\stackrel{\Xi}{\overparen{Z}}$} & \multirow{2}{*}{ Estimation using: } & \multicolumn{2}{|c|}{ FOV } \\
\hline & & in water & in tissue \\
\hline \multirow{3}{*}{ 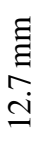 } & Theoretical & $33.3^{\circ}$ & $36.0^{\circ}$ \\
\hline & Simulation $(6 \mathrm{~dB})$ & $38.2^{\circ}$ & $37.8^{\circ}$ \\
\hline & Measurement (6dB) & $38.4^{\circ}$ & $31.6^{\circ}$ \\
\hline \multirow{3}{*}{ 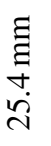 } & Theoretical & $19.8^{\circ}$ & $24.7^{\circ}$ \\
\hline & Simulation $(6 \mathrm{~dB})$ & $23.4^{\circ}$ & $24.0^{\circ}$ \\
\hline & Measurement (6dB) & $23.9^{\circ}$ & $23.4^{\circ}$ \\
\hline
\end{tabular}

Theoretical: using equation (5); Simulation: using PZFlex (Fig. 5); Measurement: using OptoSon (Fig. 6)

ultrasound beam analyzer (Onda Corporation, Sunnyvale, California, USA) was used to validate the diverging properties of the lenses in a water tank.

The contrast and spatial resolution are computed using the measures described in [9], as well as the FOV using (5).

\section{RESUlts AND Discussion}

Using (2), the focal length of the lenses with radii $12.7 \mathrm{~mm}$ and $25.4 \mathrm{~mm}$ in contact with water are $-39.04 \mathrm{~mm}$ and $-78.09 \mathrm{~mm}$. For human soft tissue, the focal lengths are $-36.21 \mathrm{~mm}$ and $-72.43 \mathrm{~mm}$. It can be noticed that the curvature of the wave fronts are smaller in water compared with human tissue. Based on the estimated focal lengths, the effect of the lenses can be represented as a virtual arc shaped elements with the appropriate curvatures for the beamforming [12].

Fig. 5 shows the simulated transmit pressure field with PZFlex (PZFlex LLC, Cupertino, CA, USA) for both lenses in contact with human soft tissue. Fig. 5 illustrates a half of the transmit beam in the lateral plane, which is symmetric across the center. The origin corresponds to the center of the transducer array. The beam profiles are generated by storing the maximum pressure at each point during propagation of the pressure wave fronts. The pressure wave fronts are produced using a one-cycled sinusoid excitation propagating through the lens material (longitudinal attenuation of $1.4 \mathrm{~dB} / \mathrm{mm}$ ) as well as the tissue $(1540 \mathrm{~m} / \mathrm{s})$ with no attenuation $(0 \mathrm{~dB} /(\mathrm{cm} \mathrm{MHz}))$ down to a $35 \mathrm{~mm}$ depth.

The theoretical, simulated, and measured beam opening angles in contact with water and human soft tissue mimicking material are listed in Table II for the lateral dimension. For the lens with radius $25.4 \mathrm{~mm}$, the simulated FOV of the $6 \mathrm{~dB}$ contour plot of the beam profile is approximately $24.0^{\circ}$ $\left(f_{\#}=-2.35\right)$. In contact with water, the simulated FOV is $23.4^{\circ}$. The simulated FOV value in contact with human soft tissue is similar to the theoretical value calculated using (2) and (4), which is $24.7^{\circ}\left(f_{\#}=-2.65\right)$. The simulated FOV in contact with human soft tissue is $-3 \%$ smaller compared with the theoretical estimations. In contact with water, the theoretical FOV is $19.8^{\circ}$. The simulated FOV in contact with water is larger than the theoretical FOV by $18 \%$ (Table II). 


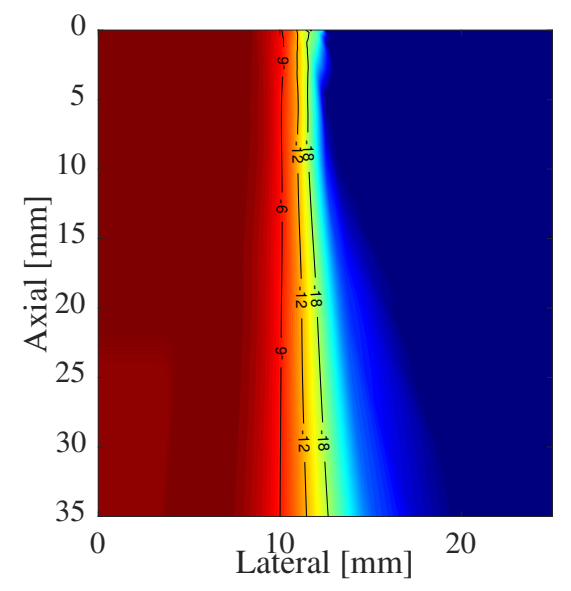

(a)

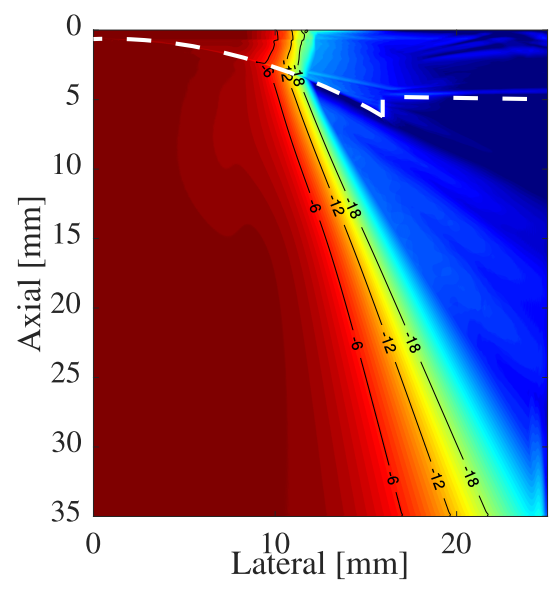

(b)

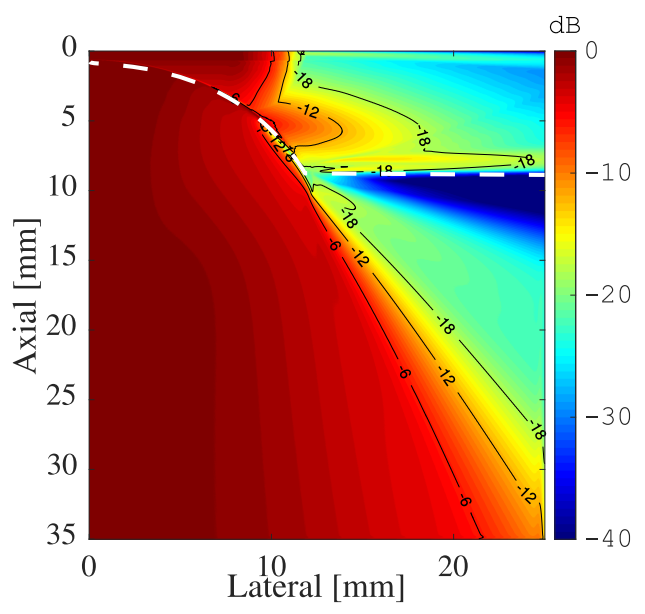

(c)

Figure 5. Simulated transmit acoustic fields with PZFlex for both lenses in contact with human soft tissue: (a) without a lens, (b) 25.4 mm radius, and (c) $12.7 \mathrm{~mm}$ radius, are shown using a $40 \mathrm{~dB}$ dynamic range. The origin corresponds to the center of the lens. The boundary of the lenses are indicated with white dashed lines.

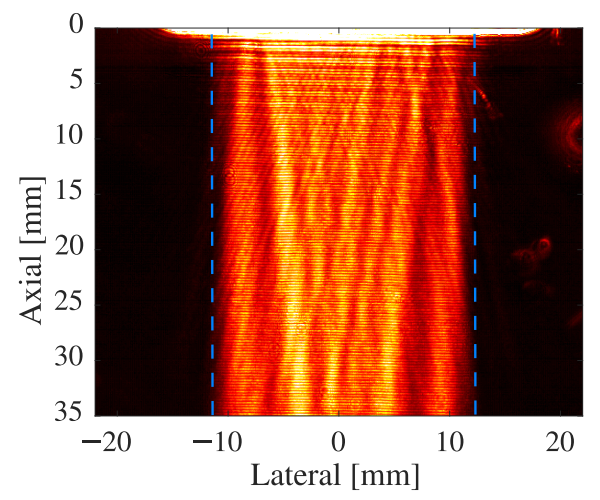

(a)

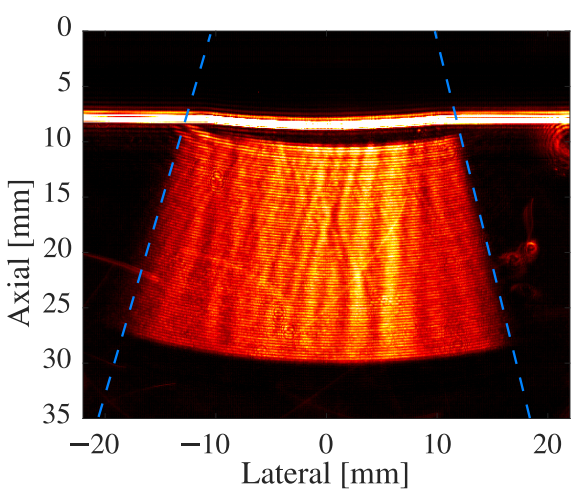

(b)

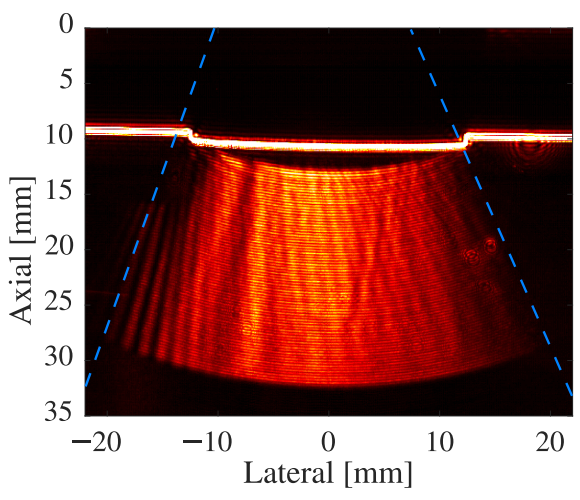

(c)

Figure 6. Measured transmitted acoustic field with an OptiSon ultrasound beam analyzer (Onda Corporation, Sunnyvale, CA, USA) for (a) the RCA array with no lens, (b) the $25.4 \mathrm{~mm}$ radius lens, and (c) the $12.7 \mathrm{~mm}$ radius lens. The images are shown with a 40 - $\mathrm{dB}$ dynamic range, where the beam at $-40 \mathrm{~dB}$ is indicated by blue dashed lines.

For the lens with radius $12.7 \mathrm{~mm}$, the simulated FOV of the $6 \mathrm{~dB}$ contour plot of the beam profile is approximately $37.8^{\circ}\left(f_{\#}=-1.46\right)$. In contact with water, the simulated FOV is $38.2^{\circ}$. The simulated FOV value in contact with human soft tissue is $36.0^{\circ}\left(f_{\#}=-1.53\right)$. The simulated FOV in contact with human soft tissue is $5 \%$ larger compared with the theoretical estimations. In contact with water, the theoretical FOV is $33.3^{\circ}$. The simulated FOV in contact with water is larger than the theoretical FOV by $15 \%$ (Table II).

The slight under estimated FOV for the $12.7 \mathrm{~mm}$ radius lens in the simulation is due to the larger attenuation through the lens material, which is not considered in (2). The estimated FOV based on the $6 \mathrm{~dB}$ contour plots should correspond to a larger FOV than the theoretical estimations, however due to the counter-effect of the roll-off apodization regions that tends to decrease the FOV, the $6 \mathrm{~dB}$ FOV is observed to be close to the theoretical estimations.

An internal reflection from the surface of the lens towards the sides and the reflections backwards the transducer is seen in the upper right corner of Fig. 5c. For a lens with a large curvature, the wave front cannot completely pass through the lens, and therefore the amplitude of the non-reflected pressure wave drops at the edges due to refraction. The amplitude drop due to refraction as well as the attenuation through the lens, decrease the SNR at the regions below the edges.

To validate the diverging properties of the lenses, Fig. 6 shows the optical projection of the density gradient generated by the acoustic pressure in water based on the Schlieren imaging concept. The data were measured using the OptiSon ultrasound beam analyzer (Onda Corporation, Sunnyvale, CA, USA). A burst of sinusoidal excitation pulses at $3 \mathrm{MHz}$ center frequency was transmitted using only one element near the center of the array. The lenses were not centered accurately on the probe during the measurement, as observed by the slight asymmetry of the beam profiles in Fig. 6b and Fig. 6c. However, by comparing the beam profiles in Fig. 6, it can be observed that using the lenses increased the FOV compared with the flat RCA array. The measured FOV for both lenses from Fig. 6 are 


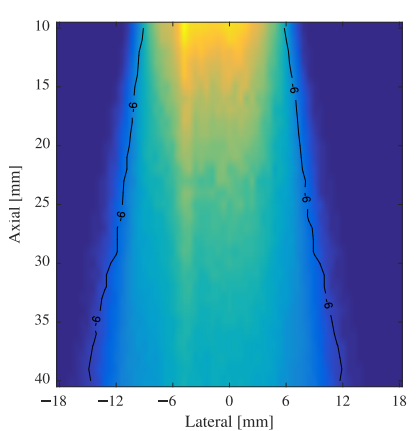

(a)

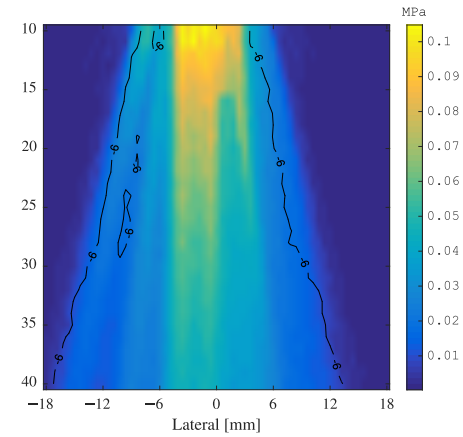

(b)
Figure 7. Measured transmitted acoustic field with hydrophone (Onda Corporation, Sunnyvale, CA, USA) in a water tank for (a) the $25.4 \mathrm{~mm}$ radius lens and (b) the $12.7 \mathrm{~mm}$ radius lens. Each point on the image represents the maximum negative pressure amplitude.

$38.4^{\circ}$ for the $12.7 \mathrm{~mm}$ radius lens and $23.9^{\circ}$ for the $25.4 \mathrm{~mm}$ radius lens, which are similar to the simulation FOVs shown in Fig. 5. The measured FOVs are larger compared with the theoretical estimation, which is due to the higher dynamic range of the optical images in Fig. 6, i.e., 40-dB. The visible FOV boundaries are indicated by blue dashed lines in Fig. 6 .

Fig. 7 shows the transmit pressure beams with both lenses in a water tank. The data was measured using a hydrophone (Onda Corporation, Sunnyvale, CA, USA) in a lateral plane. At each measurement location, the maximum negative pressure was recorded. The measured FOV for the $6 \mathrm{~dB}$ contour plots for the lenses with radii of $12.7 \mathrm{~mm}$ and $25.4 \mathrm{~mm}$ are $33.4^{\circ}$ and $18.9^{\circ}$. The measured FOVs for both lenses in contact with water are smaller than the theoretical estimation of the FOVs in contact with human soft tissue, due to their different speeds of sound in (2).

The theoretical and estimated transmit FOVs for both lenses based on simulation with PZFlex in water and tissue, as well as the measurements with hydrophone in water and derated for tissue, and also with OptiSon in water are summarized and listed in Table II.

Fig. 8 illustrates five C-planes of the volumetric pulse-echo beam patterns measured with both lenses in comparison with nolens situation using the DAS beamformation method. The iron needle faces towards the transducer, and it is imaged with the single element transmissions SAI sequence. The tip of the iron needle as point targets are located at $(x, y, z)=(0,0,43) \mathrm{mm}$ for all three cases, and at $(x, y, z)=(0,15,40) \mathrm{mm}$ for both lenses and a Hanning apodization is applied over the receive and synthesized transmit apertures before summing the low-resolution volumes in phase. Table III lists the FWHM and CR of the measured 3-D PSFs, the cross-section of which are shown in Fig. 8.

Fig. 9 illustrates three cross-planes (azimuth, elevation, and C-plane) of a wire grid phantom imaged with and without both diverging lenses. In the elevation planes in Fig. 9(d, e, f), the whole of the straight wires are not visible, which is because the reflections from the wires are inclined away from the transducer at the either ends of the wire. The secondary echoes along the axial direction associated with RCA arrays
Table III

FWHM AND CR OF MEASURED 3-D PSF 1 TO PSF 5 SHOWN IN FIG. 8

\begin{tabular}{|c|c|c|c|c|c|c|c|}
\hline & & PSF 1 & PSF 2 & PSF 3 & PSF 4 & PSF 5 & \\
\hline \multirow{3}{*}{ 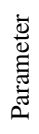 } & azimuth & 1.50 & 1.20 & 1.15 & 1.36 & 1.31 & $\mathrm{~mm}$ \\
\hline & depth & 43 & 40 & 43 & 40 & 43 & $\mathrm{~mm}$ \\
\hline & radius & 12.7 & 12.7 & 25.4 & 25.4 & n. a. & $\mathrm{mm}$ \\
\hline \multirow{3}{*}{ 乒 } & $R_{6 \mathrm{~dB}}$ & 1.50 & 1.20 & 1.15 & 1.36 & 1.31 & $\mathrm{~mm}$ \\
\hline & $R_{12 \mathrm{~dB}}$ & 2.31 & 2.0 & 1.90 & 2.30 & 2.04 & $\mathrm{~mm}$ \\
\hline & $R_{20 \mathrm{~dB}}$ & 4.30 & 5.40 & 3.15 & 4.25 & 5.25 & $\mathrm{~mm}$ \\
\hline \multirow{3}{*}{$\sum_{1}^{\sum}$} & Radial & 0.48 & 0.55 & 0.49 & 0.57 & 0.40 & $\mathrm{~mm}$ \\
\hline & Azimuth & 2.85 & 2.58 & 3.0 & 3.40 & 2.45 & $\mathrm{~mm}$ \\
\hline & Elevation & 2.88 & 3.0 & 2.97 & 3.70 & 2.51 & $\mathrm{~mm}$ \\
\hline
\end{tabular}

can be suppressed using an apodization scheme, e.g, a roll-off apodization as shown in Fig. 4. Although some secondary lobes are visible within the 40-dB dynamic range in Fig. 9(a, d), they are significantly suppressed, which depends on the fabrication of the roll-off apodization with the PZT technology. Moreover, the $12.7 \mathrm{~mm}$ radius lens does not cover the whole surface of the probe as shown in Fig. 3b, and therefore a number of secondary lobes are observed after each wire, which are not efficiently suppressed by the roll-off apodization regions in Fig. 9(c, f). However, parts of these echoes are due to reverberations from the thick corners of the lens, as well as the ghost echoes originating from either ends of the line elements. The ghost echoes are not suppressed, since the apodization regions are outside the defocusing aperture of the lens. On the other hand, the attenuation through the lens also acts as an apodization means. However, it was not enough to suppress these edge echoes in Fig. 3b. The advantage of these rolloff apodization regions is that, they do not affect the lateral resolution within the imaging FOV in comparison with other apodization methods, which tend to lower the spatial lateral resolution by widening the main lobe beam width.

Fig. 10 illustrates three cross-planes (azimuth, elevation, and C-plane) of an anechoic cyst phantom imaged with and without the diverging lenses. The phantom contains a number of anechoic cysts with radius of $5 \mathrm{~mm}$ and $2 \mathrm{~mm}$ located along a $10^{\circ}$ tilted line in the elevation plane with $20 \mathrm{~mm}$ and $10 \mathrm{~mm}$ axial spacing. Similar to previous observations, the FOV is extended compared to the flat RCA array.

A volume region of a tissue mimicking phantom with $0.5 \mathrm{~dB} /(\mathrm{cm} \mathrm{MHz})$ attenuation and no cysts was imaged 20 times for calculating the SNR. The measured SNRs for both lenses are illustrated in Fig. 11. The lens with radius $12.7 \mathrm{~mm}$ has a penetration depth of around $100 \mathrm{~mm}$ for single element transmissions, whereas the lens with radius $25.4 \mathrm{~mm}$ can penetrate down to $125 \mathrm{~mm}$ in its central axis.

Subtracting the mean value of all 20 measurements gives the actual noise in the system including that from all amplifiers and quantization noise. The time gain compensation (TGC) amplifiers have to be adjusted for each measurement situation 


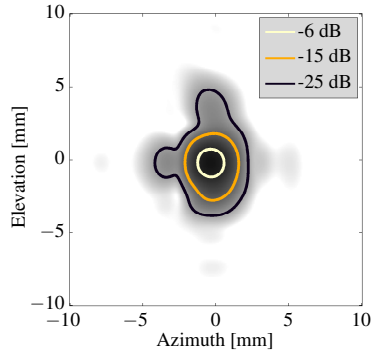

(a) $12.7 \mathrm{~mm}$ radius, $\mathrm{z}=43 \mathrm{~mm}$

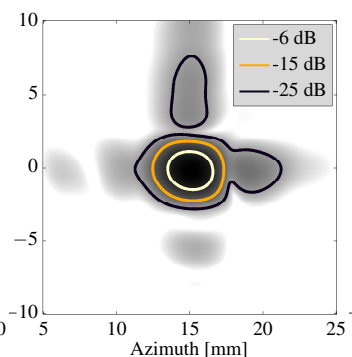

(b) $12.7 \mathrm{~mm}$ radius, $\mathrm{z}=40 \mathrm{~mm}$

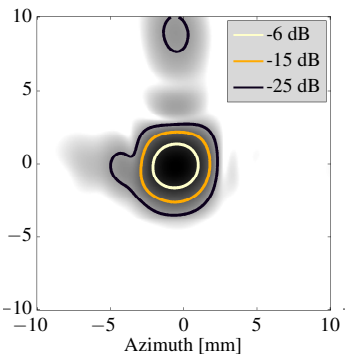

(c) $25.4 \mathrm{~mm}$ radius, $\mathrm{z}=43 \mathrm{~mm}$

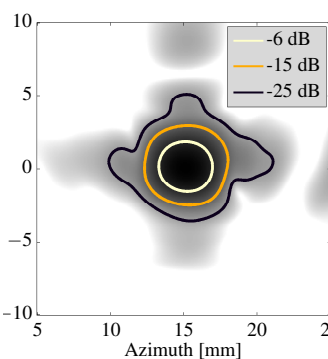

(d) $25.4 \mathrm{~mm}$ radius, $\mathrm{z}=40 \mathrm{~mm}$

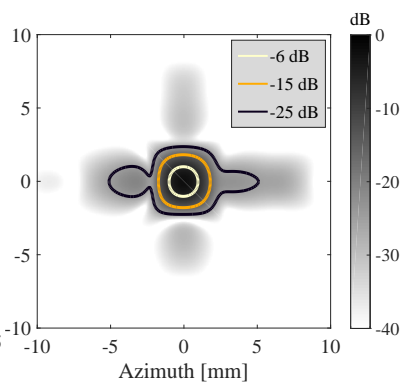

(e) no lens, $z=43 \mathrm{~mm}$

Figure 8. Five C-planes of 3-D PSFs are shown at a dynamic range of $40 \mathrm{~dB}$. The origin corresponds to the center of the transducer surface aligned with a point target positioned at $(x, y, z)=(0,0,43) \mathrm{mm}$ for (a), (c), and (e), and a point target positioned at $(x, y, z)=(0,15,40) \mathrm{mm}$ for $(\mathrm{b})$ and $(\mathrm{d})$.

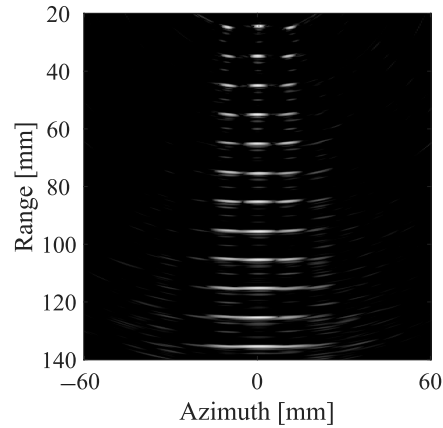

(a)

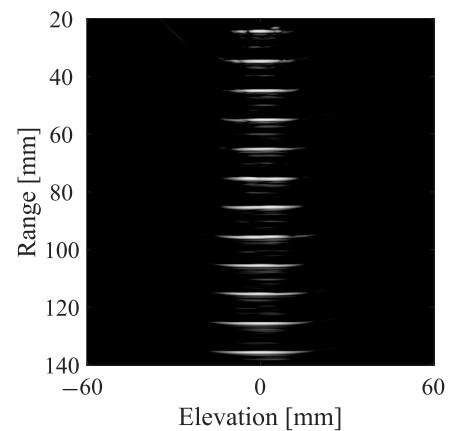

(d)

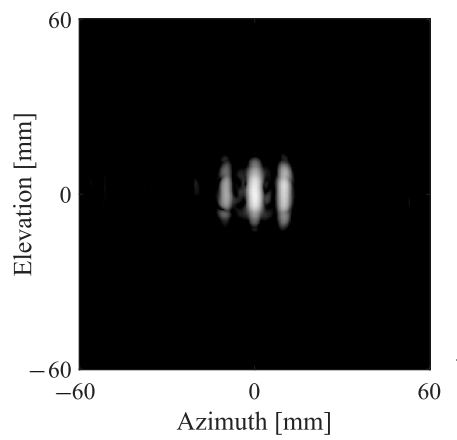

(g)

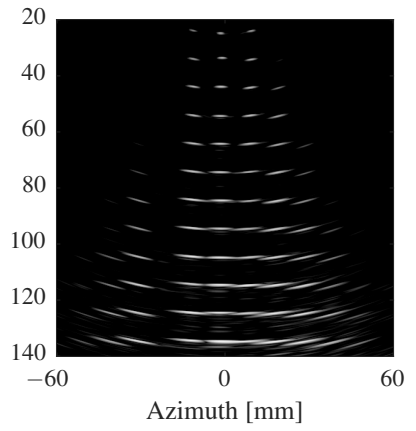

(b)

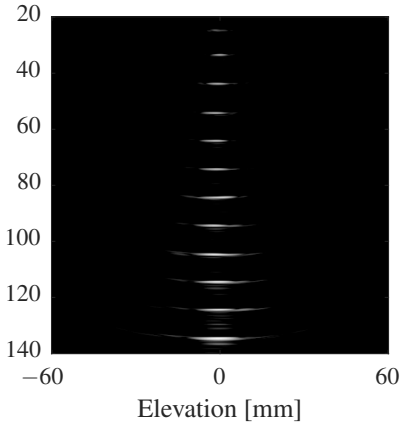

(e)

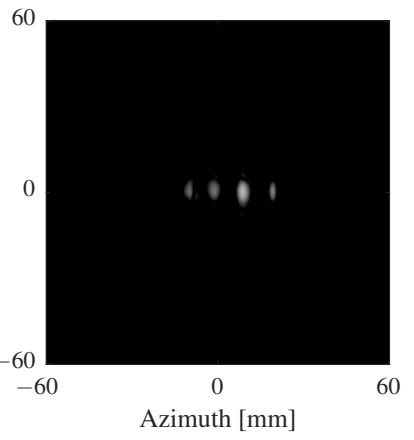

(h)

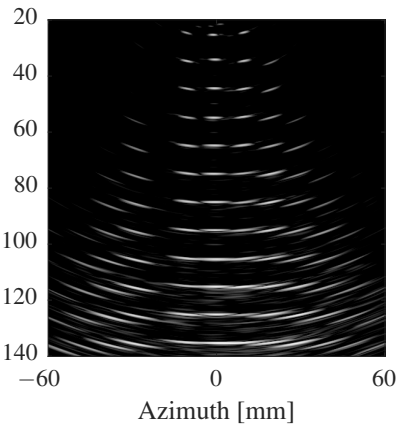

(c)

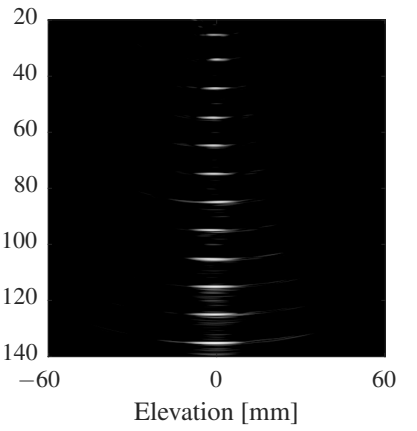

(f)

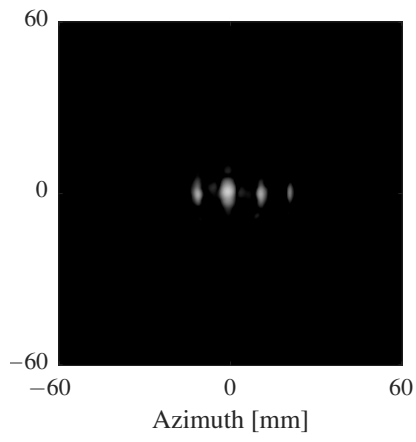

(i)

Figure 9. Three cross planes (azimuth, elevation, and C-plane) of a wire grid phantom imaged without and with both lenses are shown in a 30-dB dynamic range, (left column) without lens, (middle column) for the $25.4 \mathrm{~mm}$ radius lens, and (right column) for the $12.7 \mathrm{~mm}$ radius lens. The C-planes are at a depth of $45 \mathrm{~mm}$ and no wires are installed at $-20 \mathrm{~mm}$ lateral distance for orientation purposes. (a), (b), and (c) Azimuth plane. (d), (e), and (f) Elevation plane. (g), (h), and (i) C-plane. 


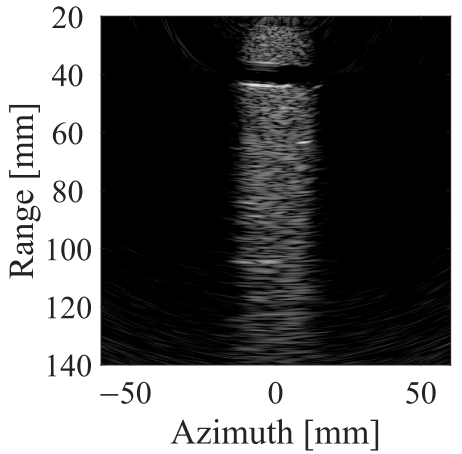

(a)

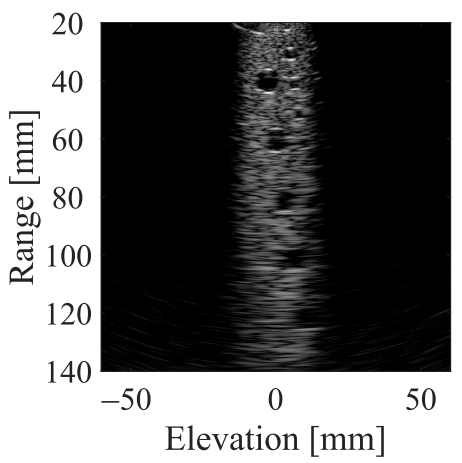

(d)

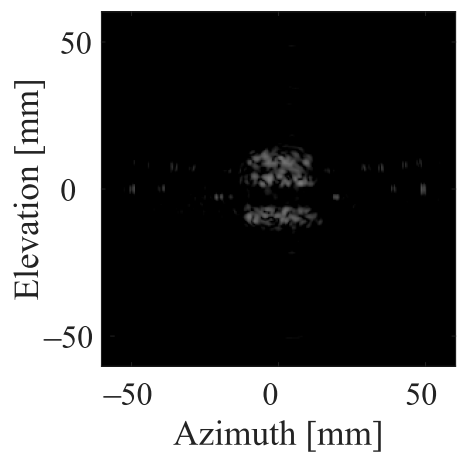

(g)

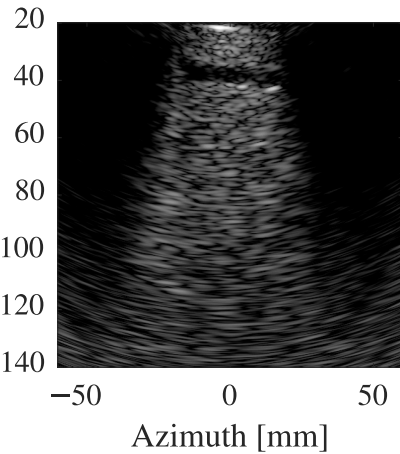

(b)

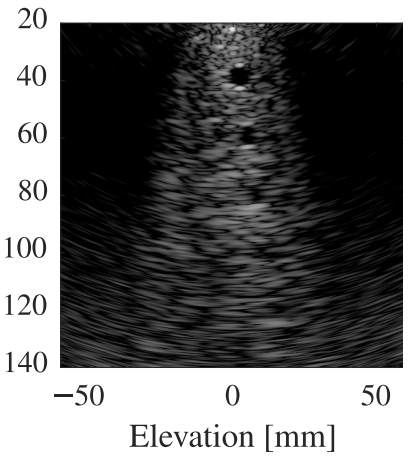

(e)

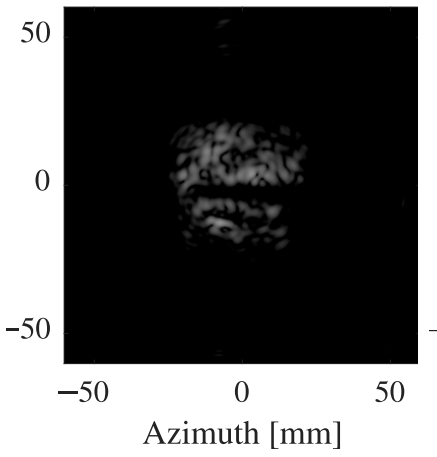

(h)

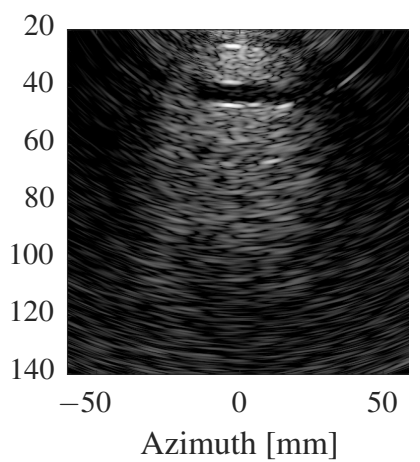

(c)

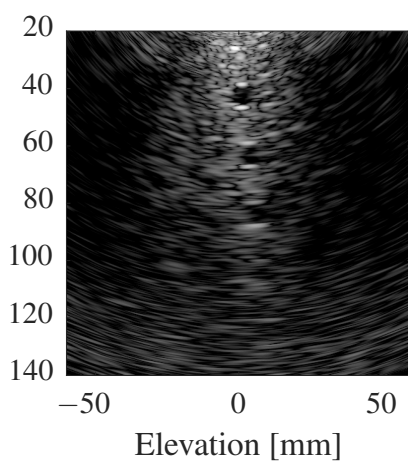

(f)

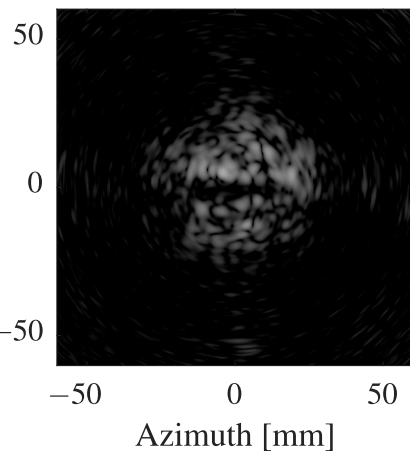

(i)

Figure 10. Three cross planes (azimuth, elevation, and C-plane) of a hollow cyst phantom imaged with both lenses are shown in a 40-dB dynamic range, (left column) without lens, (middle column) for the $25.4 \mathrm{~mm}$ radius lens, and (right column) for the $12.7 \mathrm{~mm}$ radius lens. The C-planes are at a depth of $42 \mathrm{~mm}$. (a), (b), and (c) Azimuth plane. (d), (e), and (f) Elevation plane. (g), (h), and (i) C-plane.

as the signal back is different, and a low signal would result in a high relative quantization noise, which would give an unrealizable low SNR. Using only single element in transmission lowers the SNR, however by using multiple elements in transmit by placing a virtual line source behind the array, it is possible to increase the transmit energy. On the other hand, the transmit voltage could also be scaled up by a factor to increase the SNR, which could result in a higher penetration depth, as long as it is within the acoustic safety limits.

Due to negative effects of diffraction and attenuation of the wave fronts, using a diverging lens degrades the resolution and contrast within the rectilinear FOV in front of the array, as it was shown in Fig. 8(a, c, e) and also reported in Table III. However, a diverging lens extends the FOV to a larger curvilinear region, as shown in Fig. 8(b, d), Fig. 9, and Fig. 10. Diverging the wave fronts lowers the pulse-echo energy due to diffraction compared with the conventional row-column imaging using flat arrays.

In flat RCA 2-D arrays, the amplitude of the cylindrical wave fronts as a function of depth, $r$, is proportional to $1 / \sqrt{r}$, however for the spherical wave fronts, the drop is proportional to $1 / r$. For lensed RCA 2-D arrays, the drop is proportional to $1 / \sqrt{r \cdot(r-F)}$, since the wave fronts have two different curvatures in each perpendicular dimension. For each single long-element, wave propagation in the dimension crossing the element is circular and in the dimension along the element, it is planar. Therefore, the divergence of the wave fronts in the crossing dimension is the combination of the lens effect and the circular wave diffraction pattern. For a thin lens that effect 


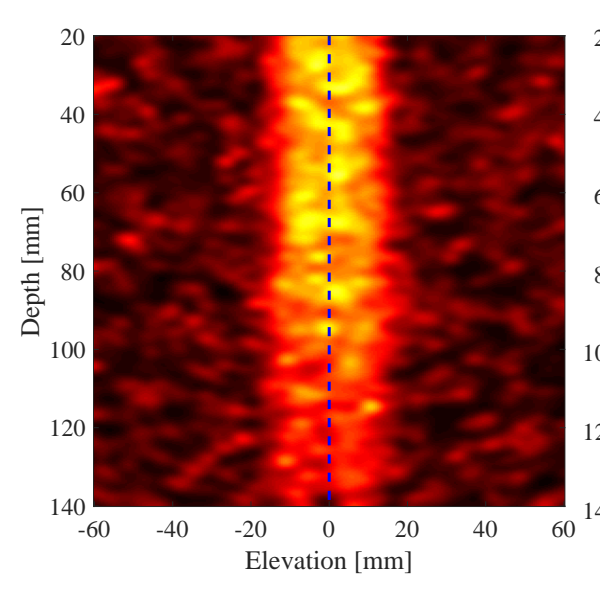

(a)

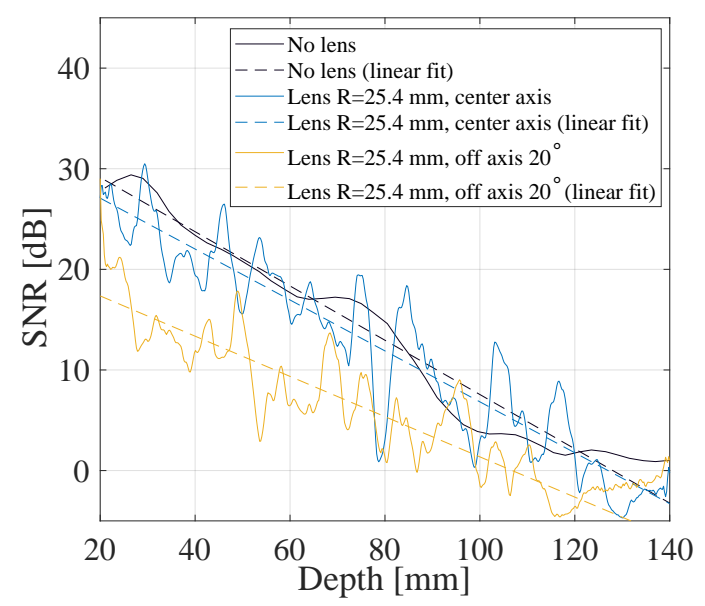

(d)

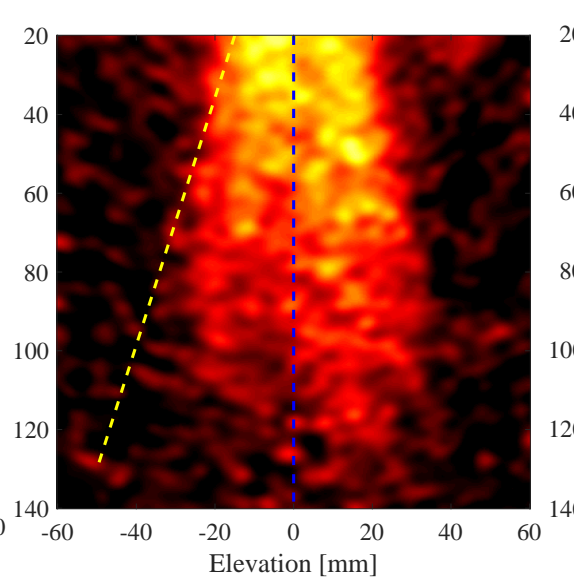

(b)

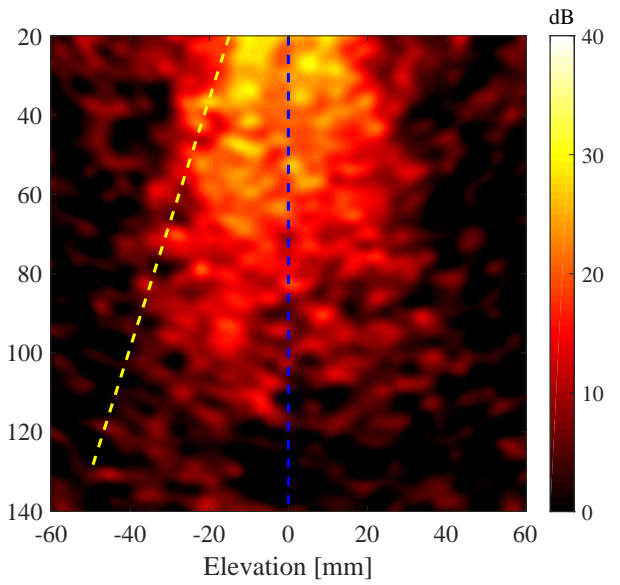

(c)

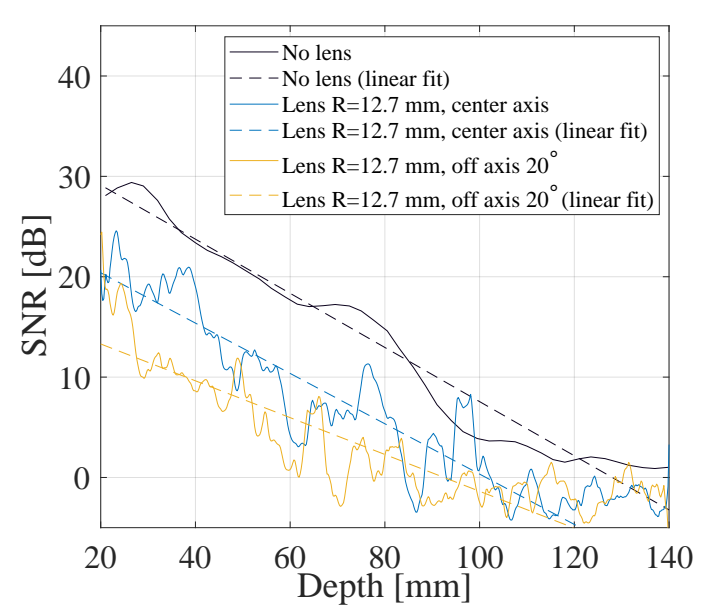

(e)

Figure 11. The SNR of the SAI sequence with and without both lenses on a tissue mimicking phantom with $0.5 \mathrm{~dB} /(\mathrm{cm} \mathrm{MHz})$ attenuation. (a), (b), and (c) are the measured SNR in elevation plane without a lens, and for the lenses with radii of $25.4 \mathrm{~mm}$ and $12.7 \mathrm{~mm}$. (d) and (e) are the SNR profiles in a region $\pm 1^{\circ}$ around the dashed lines indicated in blue and orange colors in (a), (b), and (c), respectively.

can be neglected and only the effect of the lens be considered, i.e., $\sim 1 / r$.

Furthermore, using a diverging lens lowers the amplitude due to lens attenuation. This loss of the energy can be compensated for by using all the elements in transmit and placing the transmit focus in front, over, or behind the array. Placing the focus in front of the lens, depending on the excitation length, generates two focal points, therefore calculating the time-of-flight (ToF) follows the trigonometric functions in a bispherical coordinate. On the other hand, placing the virtual line sources behind the array, the ToF calculations follows the same concept as compounding the lenses, i.e., cylindrical wave front in combination of the lens, in one dimension and no compounding in the other dimension. That is to estimate the curvature of the cylindrical wave front through a diverging lens. Placing the virtual line sources on the array can be the most straight forward approach, however all these approaches require an extensive study to better understand the propagation of the pressure waves, and therefore they are beyond the scope of this study.
In Fig. 12 three cross-planes (azimuth, elevation, and C-plane) of an anatomical abdominal phantom (Model 057A, CIRS, Norfolk, VA, USA) imaged with both diverging lenses are shown in a 40-dB dynamic range. The muscle layer, two ribs, the liver, the portal vein, and a part of the diaphragm can be seen in Fig. 12.

\section{CONCLUSION}

In this paper the curvilinear imaging performance of a $62+62$ RCA 2-D PZT array was evaluated based on phantom studies using two mountable diverging lenses. The lenses had different curvatures, one with $12.7 \mathrm{~mm}$ and the other with $25.4 \mathrm{~mm}$ radii. Using a SAI sequence with single element emissions at a time, it was possible to image down to $13 \mathrm{~cm}$ at a volume rate of $88 \mathrm{~Hz}$. The capabilities of the lenses to effectively diverge the acoustic beam were investigated using PZFlex simulation and measurement with OptiSon beam analyzer as well as pressure measurement in water bath with a hydrophone. It was shown that the rectilinear imaging FOV of the flat RCA 2-D arrays can be increased to a curvilinear imaging FOV using diverging 


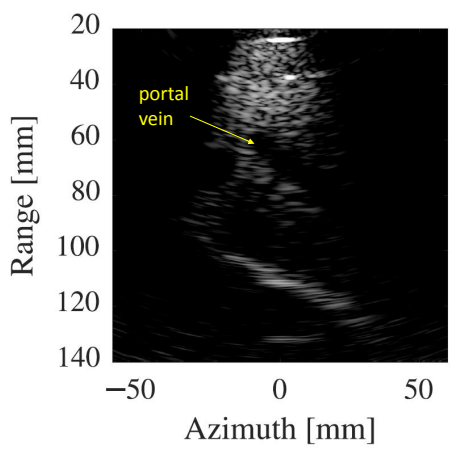

(a)

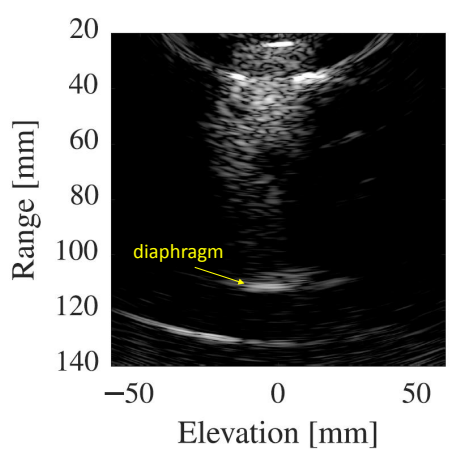

(c)

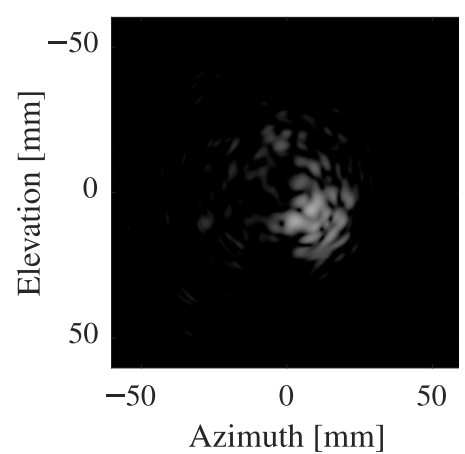

(e)

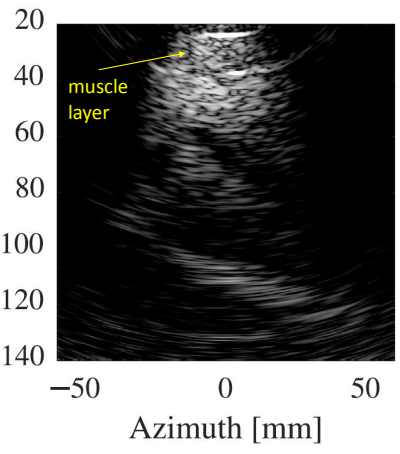

(b)

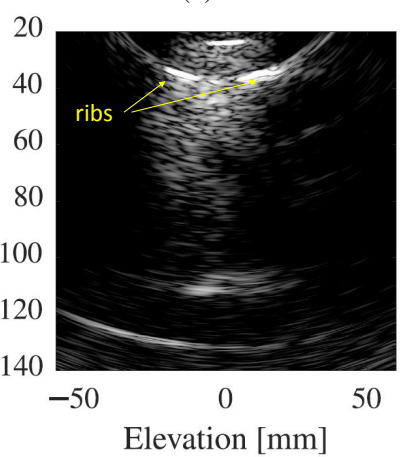

(d)

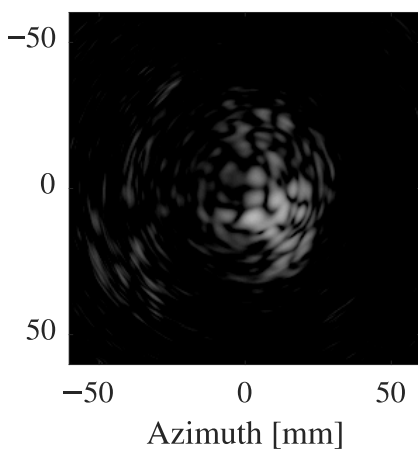

(f)
Figure 12. Three cross planes (azimuth, elevation, and C-plane) of an anatomical abdominal phantom imaged with both lenses are shown with a 40-dB dynamic range, (left column) for the $25.4 \mathrm{~mm}$ radius lens and (right column) for the $12.7 \mathrm{~mm}$ radius lens. The C-planes are at a depth of $70 \mathrm{~mm}$ (a) and (b) Azimuth plane. (c) and (d) Elevation plane. (e) and (f) C-plane.

lenses. In this study, the FOV was extended to $32^{\circ} \times 32^{\circ}$ and $24^{\circ} \times 24^{\circ}$ for lenses with radii $12.7 \mathrm{~mm}$ and $25.4 \mathrm{~mm}$ in contact with a human tissue mimicking phantom. The measured FOVs were less than $15 \%$ different from the theoretical predictions in contact with water, and differences were less than $12 \%$ in contact with a human tissue mimicking phantom.

Phantom measurement results confirm that it is also possible to perform dynamic transmit-receive focusing throughout the curvilinear FOV. Using the SAI sequence with single element emissions at a time, the penetration depth inside a human tissue mimicking phantom with $0.5 \mathrm{~dB} /(\mathrm{cm} \mathrm{MHz})$ attenuation was approximately $100 \mathrm{~mm}$ and $125 \mathrm{~mm}$ using the lenses with radii $12.7 \mathrm{~mm}$ and $25.4 \mathrm{~mm}$, respectively. Overall, having a low channel count and a large FOV offers the potential to fabricate arrays with large aperture sizes, which is important for abdominal scans with a higher spatial and contrast resolution. Thus, in comparison with the fully addressed matrix arrays, using a RCA 2-D array equipped with a diverging lens, it is possible to image 3-D volume with equipment in the price range of conventional 2-D imaging. These advantages might contribute to an increased use of real-time 3-D ultrasound imaging in medical diagnostics, and to the development of new clinical applications.

\section{ACKNOWLEDGMENT}

This work was financially supported by grant 82-2014-4 from the Danish National Advanced Technology Foundation and from BK Ultrasound ApS, Herlev, Denmark.

\section{REFERENCES}

[1] C. E. Morton and G. R. Lockwood, "Theoretical assessment of a crossed electrode 2-D array for 3-D imaging," in Proc. IEEE Ultrason. Symp. 2003, pp. 968-971.

[2] C. E. M. Démoré, A. Joyce, K. Wall, and G. Lockwood, "Real-time volume imaging using a crossed electrode array," IEEE Trans. Ultrason. Ferroelec., Freq. Contr., vol. 56, no. 6, pp. 1252-1261, 2009.

[3] C. H. Seo and J. T. Yen, "A 256 x $2562-D$ array transducer with rowcolumn addressing for 3-D rectilinear imaging," IEEE Trans. Ultrason., Ferroelec., Freq. Contr., vol. 56, no. 4, pp. 837-847, April 2009.

[4] A. Sampaleanu, P. Zhang, A. Kshirsagar, W. Moussa, and R. Zemp, "Toporthogonal-to-bottom-electrode (TOBE) CMUT arrays for 3-D ultrasound imaging." IEEE Trans. Ultrason., Ferroelec., Freq. Contr., vol. 61, no. 2, pp. 266-276, 2014.

[5] M. F. Rasmussen, T. L. Christiansen, E. V. Thomsen, and J. A. Jensen, "3-D imaging using row-column-addressed arrays with integrated apodization - Part I: Apodization design and line element beamforming," IEEE Trans. Ultrason., Ferroelec., Freq. Contr., vol. 62, no. 5, pp. 947958, 2015

[6] R. K. W. Chee, A. Sampaleanu, D. Rishi, and R. J. Zemp, "Top orthogonal to bottom electrode (TOBE) 2-D CMUT arrays for 3-D photoacoustic imaging," IEEE Trans. Ultrason., Ferroelec., Freq. Contr., vol. 61, no. 8 , pp. 1393-1395, 2014.

[7] R. J. Zemp, W. Zheng, and P. Zhang, "Feasibility of top-orthogonal-tobottom electrode (TOBE) 2D CMUT arrays for low-channel-count 3D imaging," in Proc. IEEE Ultrason. Symp., 2011, pp. 498-502.

[8] Phillips, "http://www.healthcare.philips.com," February 2015.

[9] H. Bouzari, M. Engholm, C. Beers, S. I. Nikolov, M. B. Stuart, E. V. Thomsen, and J. A. Jensen, "Imaging performance assessment of CMUT and piezoelectric row-column-addressed 2-D array probes," IEEE Trans. Ultrason., Ferroelec., Freq. Contr., p. submitted, 2016.

[10] A. I. H. Chen, L. L. P. Wong, S. Na, Z. Li, M. Macecek, and J. T. W. Yeow, "Fabrication of a curved row-column addressed capacitive micromachined ultrasonic transducer array," J. Microelectromech. S., vol. 25, no. 4, pp. 675-682, 2016.

[11] A. W. Joyce and G. R. Lockwood, "Crossed-array transducer for real-time 3D imaging," in Proc. IEEE Ultrason. Symp., 2014, pp. 2116-2120.

[12] H. Bouzari, M. Engholm, C. Beers, M. B. Stuart, S. I. Nikolov, E. V. Thomsen, and J. A. Jensen, "Curvilinear 3-D imaging using row-columnaddressed 2-D arrays with a diverging lens: Feasibility study," IEEE Trans. Ultrason., Ferroelec., Freq. Contr., vol. 64, no. 6, pp. 978-988, 2017.

[13] — "3-D imaging using row-column addressed 2-D arrays with a diverging lens: Phantom study," in Proc. IEEE Ultrason. Symp., 2017, pp. $1-4$.

[14] J. E. Greivenkamp, Field Guide to Geometrical Optics, ser. Field Guide Series. Society of Photo Optical, 2004.

[15] H. Bouzari, M. Engholm, T. L. Christiansen, C. Beers, A. Lei, M. B. Stuart, S. I. Nikolov, E. V. Thomsen, and J. A. Jensen, "Volumetric synthetic aperture imaging with a piezoelectric 2-D row-column probe," in Proc. SPIE Med. Imag., vol. 9790, 2016, pp. 1-9.

[16] J. A. Jensen, H. Holten-Lund, R. T. Nilsson, M. Hansen, U. D. Larsen, R. P. Domsten, B. G. Tomov, M. B. Stuart, S. I. Nikolov, M. J. Pihl, Y. Du, J. H. Rasmussen, and M. F. Rasmussen, "SARUS: A synthetic aperture real-time ultrasound system," IEEE Trans. Ultrason., Ferroelec. Freq. Contr., vol. 60, no. 9, pp. 1838-1852, 2013. 
[17] T. L. Christiansen, M. F. Rasmussen, J. P. Bagge, L. N. Moesner, J. A. Jensen, and E. V. Thomsen, "3-D imaging using row-column-addressed arrays with integrated apodization - part II: Transducer fabrication and experimental results," IEEE Trans. Ultrason., Ferroelec., Freq. Contr., vol. 62, no. 5, pp. 959-971, 2015.

[18] M. F. Rasmussen and J. A. Jensen, "Comparison of 3-D synthetic aperture phased array ultrasound imaging with parallel beamforming," IEEE Trans. Ultrason., Ferroelec., Freq. Contr., vol. 61, no. 10, pp. 1638-1650, 2014.

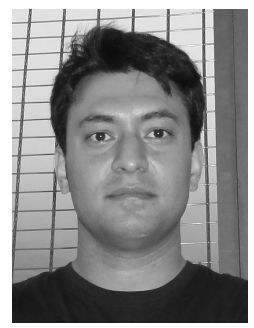

Hamed Bouzari (S'14-M'16) received the B.Sc. degree in electrical engineering from Mazandaran University, Babolsar, Iran, in 2006, the M.Sc. degree in electrical engineering from Zanjan University, Zanjan, Iran, in 2009, and the Ph.D. degree in biomedical engineering from the Technical University of Denmark, Kongens Lyngby, Denmark, in 2016.

He was a Researcher with the Austrian Academy of Sciences, Vienna, Austria, from 2010 to 2013. He is currently a Post-Doctoral Researcher with the Center for Fast Ultrasound Imaging, Technical University of Denmark. His current research interests include all facets of high volume rate 3-D ultrasonic imaging for medical applications.

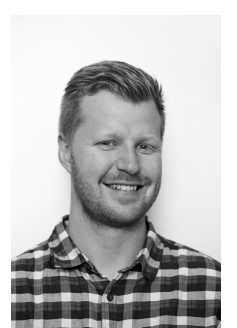

Mathias Engholm was born in Copenhagen, Denmark, in 1989. He received the B.Sc. and M.Sc. degrees in engineering physics and nanotechnology from the Technical University of Denmark, Kongens Lyngby, Denmark, in 2012 and 2015, respectively, where he is currently pursuing the Ph.D. degree, with a focus on capacitve micromachined ultrasonic transducers for 3-D imaging.

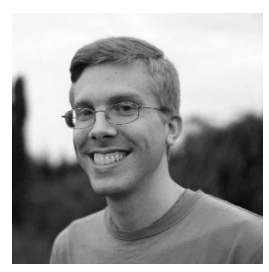

Christopher Beers received the M.S. degree in acoustics from Pennsylvania State University, State College, PA, USA, in 2007, where his thesis research explored end-element anomalies in medical ultrasound transducer arrays.

He has been with the Analogic/BK Ultrasound Group, Sound Technology Inc., State College, PA, USA, since 2007, where he has been developing transducer technology and designing commercial medical ultrasound probes.

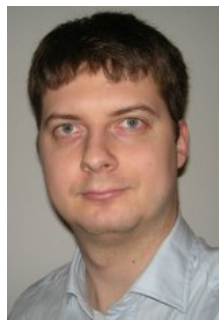

Matthias Bo Stuart (S'07-M'09) received the M.Sc. and Ph.D. degrees in computer engineering from the Technical University of Denmark, Kongens Lyngby, Denmark, in 2006 and 2010, respectively.

$\mathrm{He}$ is currently an Associate Professor with the Biomedical Engineering Group, Department of Electrical Engineering, Technical University of Denmark. His current research interests include synthetic aperture methods for both anatomical and flow imaging in 2-D and 3-D, ultrasound systems, and real-time implementations of ultrasound processing algorithms.

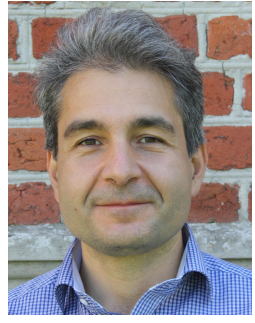

DTU.

Dr. Nikolov has been an Analogic Fellow since 2015 as recognition for his work in research and development.

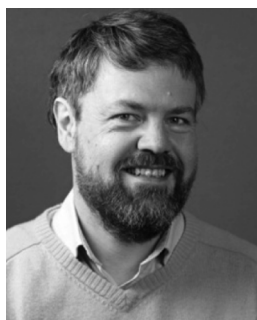

Erik Vilain Thomsen was born in Aarhus, Denmark, in 1964. He received the M.Sc. degree in physics from the University of Southern Denmark, Odense, Denmark, and the Ph.D. degree in electrical engineering from the Technical University of Denmark (DTU), Kongens Lyngby, Denmark, in 1998.

$\mathrm{He}$ is currently a Professor with DTU Nanotech, DTU, where he is also the Head of the MEMS Applied Sensors Group. He teaches classes in solid-state electronics, microtechnology, and nanofabrication and microfabrication. His current research interests include capacitive micromachined ultrasonic transducers, MEMS multisensors, biomedical devices, energy harvesting devices, and piezoelectric MEMS.

Dr. Thomsen received the AEG Electron Prize in 1995, and has received several teaching awards at DTU.

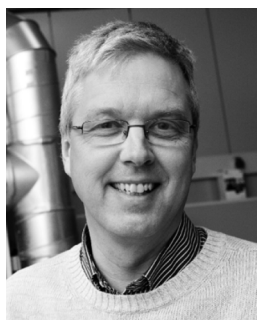

Jørgen Arendt Jensen (M'93-SM'02-F'12) received the Master of Science degree in electrical engineering in 1985 and the Ph.D. degree in 1989 , both from the Technical University of Denmark. He received the Dr.Techn. degree from the university in 1996.

Since 1993, he has been Full Professor of Biomedical Signal Processing with the Department of Electrical Engineering, Technical University of Denmark and head of the Center for Fast Ultrasound Imaging since its inauguration in 1998 . He has published more than 450 journal and conference papers on signal processing and medical ultrasound and the book Estimation of Blood Velocities Using Ultrasound (Cambridge Univ. Press), 1996. He is also the developer and maintainer of the Field II simulation program. He has been a visiting scientist at Duke University, Stanford University, and the University of Illinois at Urbana-Champaign. $\mathrm{He}$ was head of the Biomedical Engineering group from 2007 to 2010. In 2003, he was one of the founders of the biomedical engineering program in Medicine and Technology, which is a joint degree program between the Technical University of Denmark and the Faculty of Health and Medical Sciences at the University of Copenhagen. The degree is one of the most sought-after engineering degrees in Denmark. He was chairman of the study board from 2003 to 2010 and Adjunct Professor with the University of Copenhagen from 2005 to 2010 He has given a number of short courses on simulation, synthetic aperture imaging, and flow estimation at international scientific conferences and teaches biomedical signal processing and medical imaging at the Technical University of Denmark. His research is centered around simulation of ultrasound imaging, synthetic aperture imaging, vector blood flow estimation, and construction of ultrasound research systems.

Dr. Jensen has given more than 60 invited talks at international meetings and received several awards for his research. 